\title{
Calculations on Mode Eigenvalues in a Corrugated Waveguide with Varying Diameter and Corrugation Depth
}

\author{
Daniel Haas ${ }^{1}$ (D) Manfred Thumm ${ }^{1,2}$ (D) John Jelonnek ${ }^{1,2}$
}

Received: 5 August 2020 / Accepted: 7 April 2021 / Published online: 23 April 2021

(C) The Author(s) 2021

\begin{abstract}
The present paper addresses numerical calculations on the eigenvalues of hybrid modes in corrugated circular waveguides with varying diameter and corrugation depth. Such calculations are essential for the numerical optimization of advanced mode converters and diameter tapers for future low-loss high-power microwave applications, like broadband high-power radar sensors for space debris observation in low earth orbit (LEO). Corresponding mode converters and diameter tapers may be synthesized based on coupled mode theory. Of particular importance here is the ability to consider varying mode eigenvalues along the perturbed waveguide. The procedure presented here is able to consider arbitrary variations of the corrugation depth as well as the waveguide diameter and therefore is highly flexible. The required computational effort is low. Limitations of the method are discussed.
\end{abstract}

Keywords High-power microwaves · Corrugated waveguides $\cdot$ Hybrid modes $\cdot$ Coupled mode theory $\cdot$ Mode converters $\cdot$ Diameter tapers

\section{Introduction}

As already predicted in the late 1970s [1], space debris become a major issue for the use of satellites $[2,3]$. In particular, the amount of space debris in low earth orbit (LEO) is increasing rapidly [3]. To detect and map space debris, high performance radar sensors can be used $[3,4]$. Due to the enormous progress in the field of high-power microwave technology $[5,6]$, corresponding radar sensors can also be operated in high frequency bands such as the W-band $[7,8]$. Due to the high bandwidth available there, very high

Daniel Haas

daniel.haas@kit.edu

1 IHM, Karlsruhe Institute of Technology (KIT), Kaiserstr. 12, D-76131 Karlsruhe, Germany

2 IHE, Karlsruhe Institute of Technology (KIT), Kaiserstr. 12, D-76131 Karlsruhe, Germany 
resolutions can be achieved [7-9]. In near future, even W-band transmission powers in the range of $100 \mathrm{~kW}$ may be achieved [10]. To realize a W-band radar sensor with such a high transmission power, a suitable high-power amplifier as well as an appropriate transmission line is required. The transmission line connects the output of the highpower amplifier with the antenna feed. Due to the high power, oversized transmission lines are required. A suitable transmission mode is the $\mathrm{HE}_{11}$ hybrid mode in a corrugated circular waveguide [11-13]. Low ohmic loss, small mode conversion, and low-sidelobe radiation patterns can be achieved [11-13]. Important components of an overmoded transmission line are miter bends [14, 15], polarizers [16, 17], rotary joints [18], and waveguide diameter tapers $[19,20]$. For design verification of such components, low power measurements are required. Here, the fundamental $\mathrm{TE}_{10}$ mode within a rectangular waveguide, provided by a microwave extension module [21] of a Network Analyzer (NWA), has to be converted into the $\mathrm{HE}_{11}$ hybrid mode within a highly oversized corrugated circular waveguide. In a simple way, this may be realized by a corrugated horn antenna $[22,23]$, illuminating the aperture of a highly overmoded circular waveguide $[24,25]$. However, since the component behavior under test may be sensitive in respect to the mode content $[14,15]$, a mode purity as best as possible is required, within the considered frequency range. A concept based on a corrugated horn is limited in respect of the mode purity $[24,25]$. Therefore, a waveguide mode converter is preferred here, which may attain a significant better mode purity [24]. Such a mode converter may consist of (1) commercial rectangular $\mathrm{TE}_{10} \mapsto$ circular $\mathrm{TE}_{11}$ transition and (2) customized $\mathrm{TE}_{11} \mapsto \mathrm{HE}_{11}$ mode converter, based on tapered variation of the corrugation depth [26, 27], integrated within a waveguide diameter up-taper.

For the numerical synthesis of such an advanced mode converter with broadband frequency behavior, coupled mode theory [28-30] may be applied. However, in calculations on a perturbed corrugated waveguide, varying eigenvalues are of particular importance to consider. The present paper addresses fast numerical calculations on eigenvalues of a corrugated waveguide with varying diameter and corrugation depth. To the best knowledge of the authors, similar calculations along an arbitrary perturbation were not published yet.

The paper is organized as follows: Section 2 introduced the coupled mode formalism, able to calculate mode conversion within multimode waveguides. In Section 3, fundamentals of corrugated waveguides and their eigenvalues are discussed. Section 4 addresses numerical calculations on the eigenvalues of a corrugated waveguide with varying diameter and corrugation depth. Section 5 closes with the conclusion and an outlook for future research activities.

\section{Coupled Mode Theory}

The principle concept of the coupled mode theory is to expand an electromagnetic field within a perturbed waveguide as superposition of eigenmodes of a uniform reference waveguide, with the same cross section [28-30]. Neglecting ohmic wall losses and backward travelling waves due to reflections (highly oversized waveguide) as well as restricting to adiabatically varying waveguide parameters (characteristic length of variation large compared to the wavelength), the corresponding mode amplitudes satisfy the first-order differential equation [28-30]: 


$$
\frac{\mathrm{d}}{\mathrm{d} z} I_{n}(z)=-j \beta_{n} \cdot I_{n}(z)+\sum_{m}^{n \neq m} \mathrm{~K}_{n m} \cdot I_{m}(z)
$$

with $I_{n}(z)$ as complex mode amplitude at the position $z, \beta_{n}$ as corresponding phase constant, and $\mathrm{K}_{n m}$ as coupling coefficient [30-32] between the $n$th and $m$ th eigenmode of the uniform reference waveguide. To eliminate fast varying phase terms, the substitution $I_{n}(z)=A_{n}(z) \cdot \exp \left[-j \beta_{n} \cdot z\right]$ is introduced with $A_{n}(z)$ as slowly varying complex mode amplitude. Using the Runge-Kutta integration method [33], such a substitution reduces the required step width and therefore the required computational effort. It follows:

$$
\frac{\mathrm{d}}{\mathrm{d} z} \mathrm{~A}_{n}(z)=\sum_{m}^{n \neq m} \mathrm{~K}_{n m} \cdot \exp [-j \Delta \beta \cdot z] \cdot A_{m}(z)
$$

with

$$
\Delta \beta=\beta_{n}-\beta_{m}
$$

In general, the exponent in Eq. (2) has to be substituted by [11, 34]:

$$
-j \int_{0}^{L} \Delta \beta \mathrm{d} z
$$

with $L$ as the perturbation length. This allows consideration of varying phase constants, for example, caused by a varying waveguide diameter.

In matrix representation, Eq. (2) reads:

$$
\frac{\mathrm{d}}{\mathrm{d} z} \mathbf{\Upsilon}(z)=\mathbf{H} \cdot \mathbf{\Upsilon}(z)
$$

with

$$
\Upsilon(z)=\left(\begin{array}{c}
A_{1}(z) \\
A_{2}(z) \\
\vdots \\
A_{N}(z)
\end{array}\right)
$$

and

$$
h_{i j}=\left\{\begin{array}{cl}
0 & \text { for } i=j \\
\mathrm{~K}_{n m} \cdot \exp \left[-j \int_{0}^{L}\left(\beta_{i}-\beta_{j}\right) \mathrm{d} z\right] & \text { for } i \neq j
\end{array}\right.
$$

as the coefficients of the coupling matrix $\mathbf{H}$.

Equations (5)-(7) show that knowledge about varying phase constants is of particular importance to control mode conversion within a perturbed waveguide. This results in the particular importance of varying mode eigenvalues since the phase constant depends on the waveguide diameter as well as on the mode eigenvalue [11]:

$$
\beta=k \cdot \sqrt{1-\left(\frac{X}{k a}\right)^{2}}
$$

with $a$ as the waveguide diameter, $k=2 \pi / \lambda$ as the free-space wavenumber, and $X$ as the mode eigenvalue. 


\section{Corrugated Waveguide}

Figure 1 shows a simplified sketch of a corrugated waveguide with the radius $a$, the corrugation period $c$, the slot width $b$, and the corrugation depth $d$.

Typically, hybrid modes of a corrugated waveguide are referred according to the balanced hybrid mode condition [22]:

$$
\vartheta_{m}(X)= \pm m
$$

with

$$
\vartheta_{m}(X)=X \cdot \frac{J_{m}^{\prime}(X)}{J_{m}(X)}
$$

The function $J_{m}(x)$ is the Bessel function of the first kind and the order $m$. The function $J_{m}^{\prime}(x)$ is its first derivation in respect of $x$. For the $n$th root and $+/-, \mathrm{HE}_{m n} / \mathrm{EH}_{m n}$ modes occur [22, 35]. Equation (9) is valid for a highly oversized waveguide $(k a=2 \pi \cdot a / \lambda \mapsto \infty)$ having a longitudinal wall surface impedance $Z_{s}=-E_{z} / H_{\varphi} \neq 0$. For $k a \neq \infty$, the surface-impedance model $[11,22,31,32]$, which assumes a uniform surface impedance, is widely used. Corresponding calculations lead to [35]:

$$
\vartheta_{m}(X)-\frac{(m \tilde{\beta})^{2}}{\vartheta_{m}(X)}=\left(\frac{X}{k a}\right)^{2} \cdot S_{m}(k a, k(a+d))
$$

with

$$
\begin{aligned}
\widetilde{\beta} & =\beta / k=\sqrt{1-\left(\frac{X}{k a}\right)^{2}} \\
S_{m}(x, y) & =x \cdot \frac{J_{m}^{\prime}(x) Y_{m}(y)-J_{m}(y) Y_{m}^{\prime}(x)}{J_{m}(x) Y_{m}(y)-J_{m}(y) Y_{m}(x)}
\end{aligned}
$$

The function $Y_{m}(x)$ is the Bessel function of the second kind and the order $m$, and $Y_{m}^{\prime}(x)$ is its first derivation in respect of $x$.

In principle, Eq. (11) is derived as [35-39] (1) calculation of the field components for $r \leq a$ as superposition of transversal electric (TE) and transversal magnetic (TM) field components,

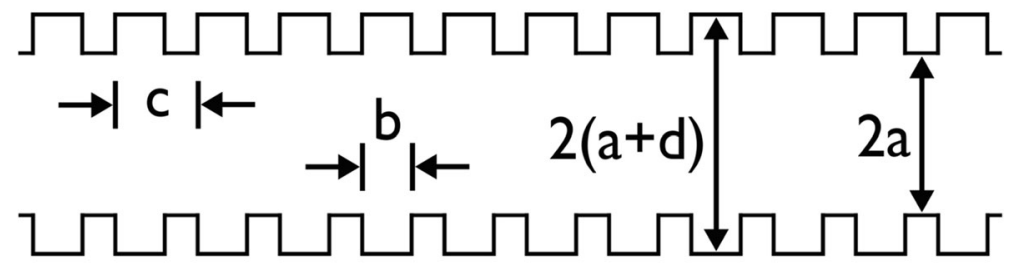

Fig. 1 Corrugated waveguide with the waveguide radius $a$, the corrugation period $c$, the slot width $b$, and the corrugation depth $d$ 
(2) calculation of the field components for $a<r<(a+d)$ as a pure TM mode with $E_{z}=$ const., and (3) matching the field components at $r=a$.

A pure TM mode within the corrugation requires $b<\lambda / 2$. In this way, a radial TE mode cannot be supported, and no Bragg reflection $[40,41]$ occurs $[42,43]$. The longitudinal surface impedance $Z_{s}$ at $r=a$ can be represented as [35, 44]:

$$
Z_{s}=-j Z_{0} \cdot \frac{k a}{S_{m}(k a, k(a+d))} \approx j Z_{0} \tan (k d)
$$

For $k a \mapsto \infty$ and $Z_{s} \neq 0$, Eq. (11) merges into Eq. (9).

Note that the surface-impedance model neglects space harmonics $[45,46]$ and therefore is restricted to $c \approx b$ (thin corrugation ridges) and $b \ll \lambda / 2$ (large number of corrugation slots per wavelength) [22, 23, 35-39]. In more general calculations, space harmonics within $r \leq a$ as well as $a<r \leq(a+d)$ have to be considered. Corresponding calculations lead to rather elaborate equations [22] which are out of scope of the present paper. Simplified equations result for the restriction to $K$ space harmonics within $r \leq a$. Corresponding calculations lead to [22]:

$$
\frac{b}{c} \cdot \sum_{N=-K}^{K}\left(\frac{1}{\eta}\right)^{2} \cdot\left[\vartheta_{m}(\eta)-\frac{\left(m \widetilde{\beta}_{N}\right)^{2}}{\vartheta_{m}(\eta)}\right] \cdot \frac{\sin \left(\beta_{N} \cdot \frac{b}{2}\right)}{\left(\beta_{N} \cdot \frac{b}{2}\right)}=\frac{S_{m}(k a, k(a+d))}{(k a)^{2}}
$$

In principle, the derivation of Eq. (14) is identical to the mode matched procedure introduced for the surface-impedance model. But now, for $r \leq a$, space harmonics are considered with $\beta_{N}=\beta+N \cdot 2 \pi / c, \widetilde{\beta}_{N}=\beta_{N} / k$, and $\eta=a \cdot \sqrt{k^{2}-\beta_{N}^{2}} \quad[22,45,46]$. For $K=0$ (fundamental space harmonic), $c \approx b$, and $b \ll \lambda / 2$, Eq. (14) merges into Eq. (11).

Figure 2 compares calculated eigenvalues for $m=1\left(\mathrm{HE}_{1 n} / \mathrm{EH}_{1 n}\right.$ modes $)$ and $X \in[0,7]$, based on Eq. (14) with $K=1$ (first spatial harmonic) and the surface-impedance model, given in Eq. (11). The blue data points are calculated using the surface impedance model. The yellow data points are calculated with $k a=10, b=0.1 \cdot \lambda / 2$, and $c=1.1 \cdot b$, fulfilling the terms and conditions of the surface-impedance model $(c \approx b, b \ll \lambda / 2)$, whereas the brown data points are calculated with $k a=10, b=0.4 \cdot \lambda / 2$, and $c=2 \cdot b$, violating the terms and conditions of the surface-impedance model. Figure 2 shows good agreement of the surface-impedance model and Eq. (14), as long as those terms and conditions are fulfilled. Otherwise, significant deviations occur.

The curves in Fig. 2 were calculated using the Newton-Raphson method, addressed in Section 4.1. Calculations with $K=1$ and $K=2$ revealed that the consideration of an increasing number of space harmonics leads just to small variations compared with the calculations based on the first space harmonic.

In the following, the consideration is restricted to the surface-impedance model. The explicit corrugation geometry is omitted here which leads to a more descriptive representation of the principle procedure. However, this requires that the terms and conditions of the surfaceimpedance model $(c \approx b, b \ll \lambda / 2)$ are fulfilled (see Fig. 2$)$. In the case, that they cannot be fulfilled; only Eq. (11) must be replaced by Eq. (14). 


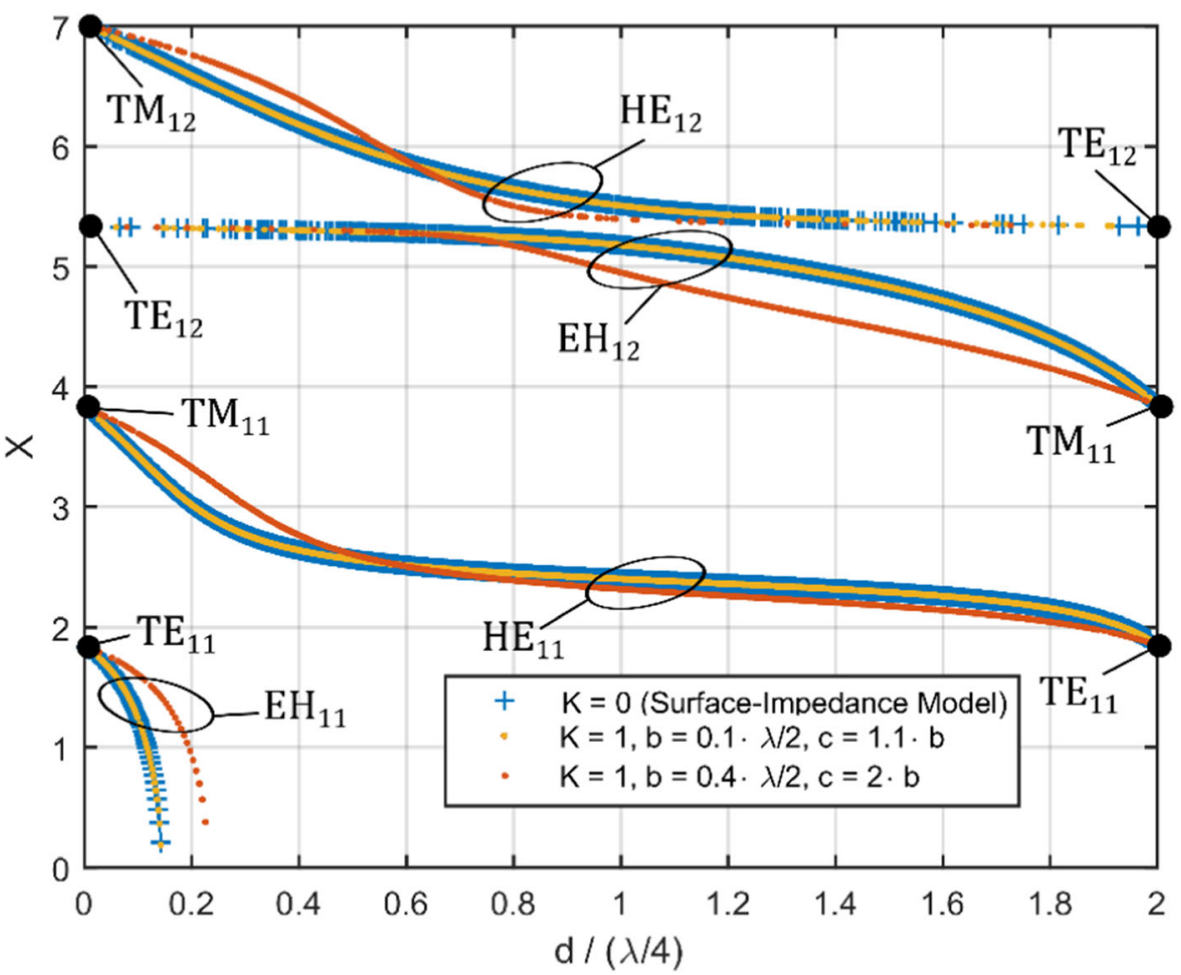

Fig. 2 Calculated eigenvalues for $m=1\left(\mathrm{HE}_{1 n} / \mathrm{EH}_{1 n}\right.$ modes $), X \in[0,7]$, and $k a=10$ for varying corrugation depth $d$, considering the first space harmonic ( $K=1$, yellow and brown curves) and the surface-impedance model (blue curve)

\section{Numerical Calculations}

To calculate the variation of the eigenvalues along an arbitrary perturbation, Eq. (11) is solved numerically. In Subsection 4.1, the Newton-Raphson method is introduced, which here is used for numerical calculations. Subsection 4.2 addresses corresponding results.

\subsection{Newton-Raphson Method}

The principle concept of the Newton-Raphson method is to linearize the considered function $f(x)$ at a starting point $x_{0}$ and use those roots as improved starting point of a second iteration $[47,48]$. It follows:

$$
x_{n+1}=x_{n}+\Delta x
$$

with

$$
\Delta x=-\frac{f\left(x_{n}\right)}{f^{\prime}\left(x_{n}\right)}
$$

The iteration is aborted when the rate of change of the approximated solution falls below a specified value. 
For numerical calculations, the central difference quotient can be used [49]:

$$
f^{\prime}(x) \approx \frac{f(x+h)-f(x-h)}{2 h}
$$

To avoid tripping due to a too small $h$ and a not sufficient accuracy through a too large $h$, $h=\sqrt{\text { eps }}$ is used here, with eps as floating point relative accuracy (for double precision: eps $=2^{-52}$ ) [50].

To find all solutions of interest, the Newton-Raphson method is calculated multiple times with random initialization within the range of interest.

\subsection{Results}

Figure 3 shows the calculated eigenvalues for $m=1\left(\mathrm{HE}_{1 n} / \mathrm{EH}_{1 n}\right.$ modes $), X \in[0,7]$, and very large $k a=1000$ ( $\widehat{=} \infty$, blue curves), as well as for $k a=10$ (brown curves) in dependence of the corrugation depth $d \in[0, \lambda / 2]$. For $k a \mapsto \infty$, the eigenvalues are close to constant. For moderate $k a$, the eigenvalues depend strongly on the corrugation depth. However, for $d \approx \lambda / 4$, in each case, broadband frequency behavior is shown, with a relative bandwidth far beyond $10 \%$. For $d \mapsto 0$ or $\lambda / 2$, Eq. (13) gives $Z_{s}=0$, and the $\mathrm{HE}_{m n} / \mathrm{EH}_{m n}$ modes merge into ordinary $\mathrm{TE}_{m n} /$ $\mathrm{TM}_{m n}$ modes [51,52]. As shown in Fig. 3, this transition is abrupt at large $k a$. For a $\mathrm{TE}_{11} \mapsto$ $\mathrm{HE}_{11}$ mode converter, discussed in Section 1, this is critical in respect of parasitic mode conversion $[27,51,52]$.

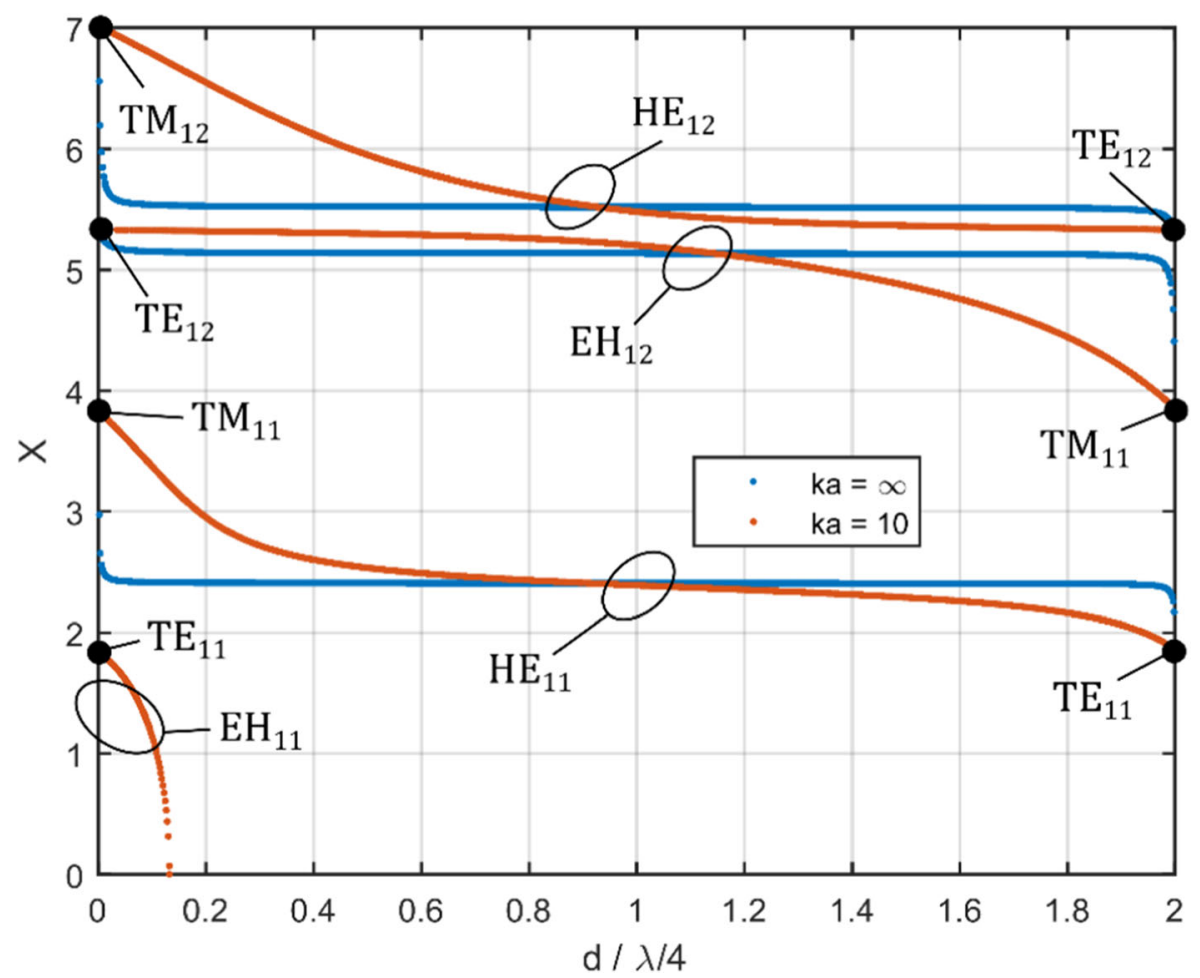

Fig. 3 Eigenvalues for $k a \mapsto \infty$ and $k a=10$ in dependence of the normalized corrugation depth $d$ 
To ensure a high mode purity, a customized variation as well on the corrugation depth [26, $27,51,52]$ as the waveguide diameter may be used. However, this requires the ability to calculate the variation of the eigenvalues along an arbitrary perturbation with low computational effort. This is possible with the presented procedure here. As example, Fig. 4 shows numerical calculations for $m=1\left(\mathrm{HE}_{1 n} / \mathrm{EH}_{1 n}\right.$ modes), $X \in[0,7]$, and $k a=50$ (blue color) along a tapered variation of the corrugation depth (brown color), with:

$$
\frac{\mathrm{d}}{\mathrm{d} z} d(z) \propto \sin ^{2}\left(\frac{\pi}{L} z\right)
$$

Figure 4 shows that due to the tapered variation of the corrugation depth, the strong variation at $d \mapsto 0$ or $\lambda / 2$ could be smoothened. The eigenvalues change slowly along the perturbation, which leads to low parasitic mode conversion. For a $\mathrm{TE}_{11} \mapsto \mathrm{HE}_{11}$ mode converter, discussed in Section 1 , this is essential $[26,27,51,52]$. For a numerical synthesis, an even more optimized tapering of the corrugation depth may be applied. However, this is out of scope of the present paper. For comparison, Fig. 5 shows the eigenvalues along a perturbation with $k a=50$ and a constant rate of change (linear progression).

\section{Conclusion}

The present paper addresses fast numerical calculations on the eigenvalues of hybrid modes in corrugated circular waveguides with arbitrary perturbation of the waveguide diameter and the corrugation depth, in case that the surface-impedance model is valid.

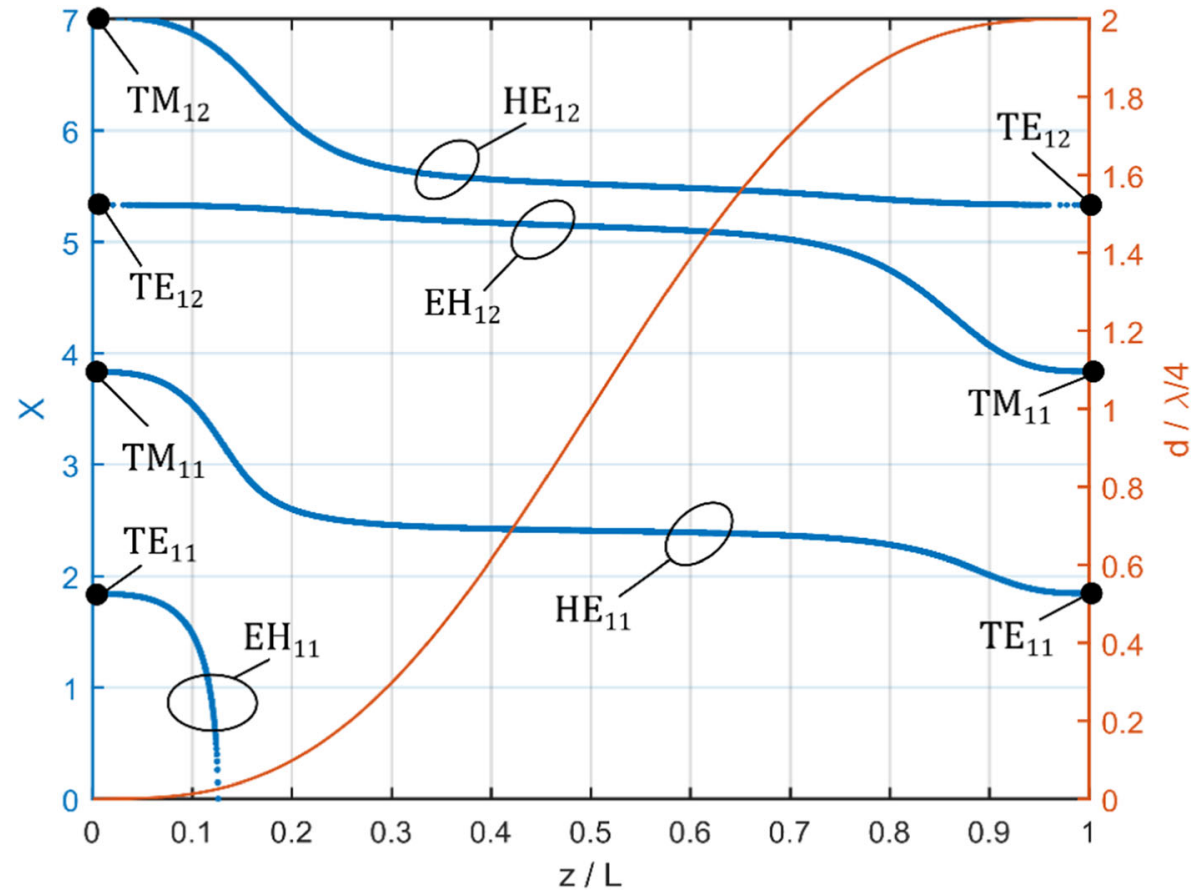

Fig. 4 Eigenvalues for $k a=50$ and a tapered rate of change of the varying corrugation depth $d$ 


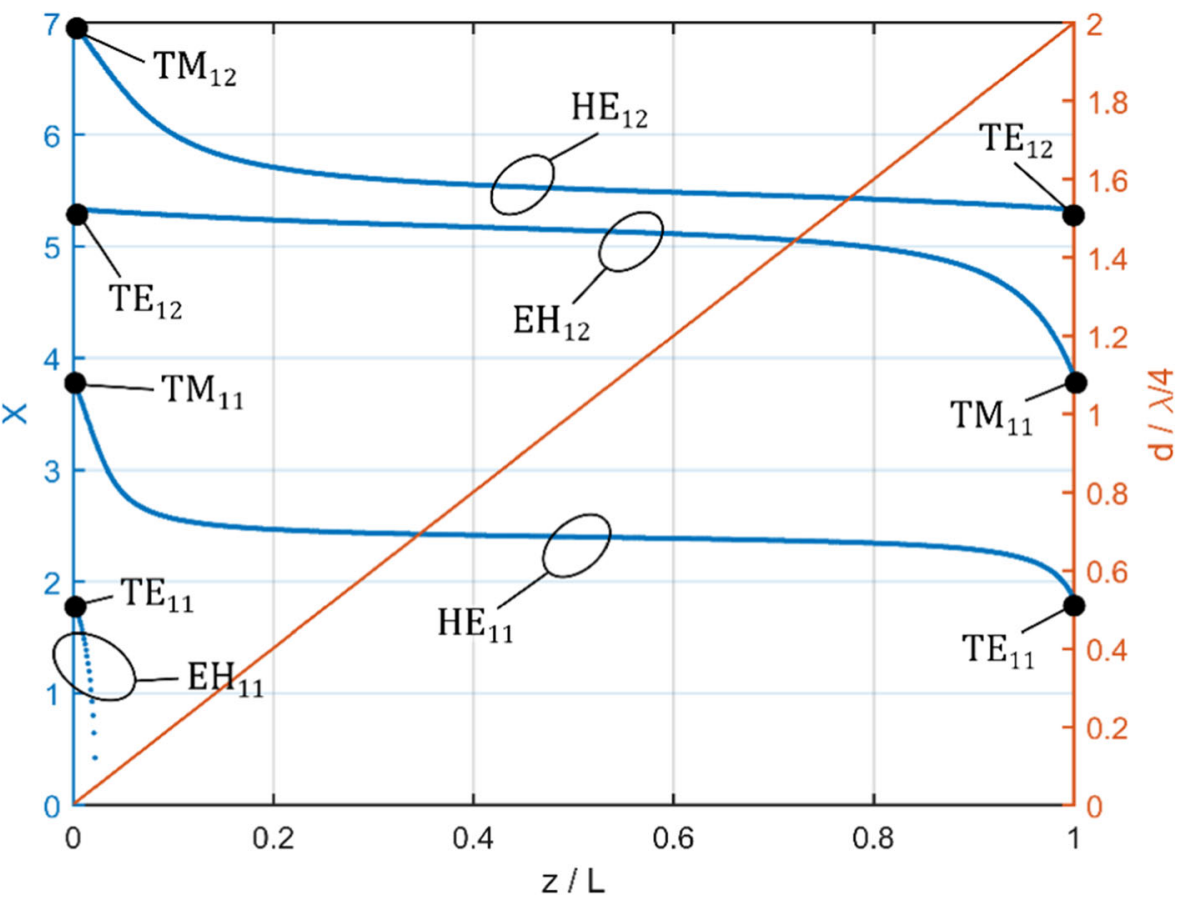

Fig. 5 Eigenvalues for $k a=50$ and a constant rate of change of the varying corrugation depth $d$

Such calculations are essential for the synthesis of optimized mode converters and diameter tapers within corrugated waveguides.

Future research activities will address the embedding of the outlined procedure into the numerical synthesis of broadband corrugated waveguide mode converters and diameter tapers. Such passive microwave components will be essential for future broadband high-power microwave applications.

Funding Open Access funding enabled and organized by Projekt DEAL.

Open Access This article is licensed under a Creative Commons Attribution 4.0 International License, which permits use, sharing, adaptation, distribution and reproduction in any medium or format, as long as you give appropriate credit to the original author(s) and the source, provide a link to the Creative Commons licence, and indicate if changes were made. The images or other third party material in this article are included in the article's Creative Commons licence, unless indicated otherwise in a credit line to the material. If material is not included in the article's Creative Commons licence and your intended use is not permitted by statutory regulation or exceeds the permitted use, you will need to obtain permission directly from the copyright holder. To view a copy of this licence, visit http://creativecommons.org/licenses/by/4.0/.

\section{References}

1. D. Kessler and B. Cour-Palais: "Collision Frequency of Artificial Satellites: The Creation of a Debris Belt", Journal of Geophysical Research, Vol. 83, No. A6, 2637-2646 (1978).

2. M. Williamson: "Space Junk makes an Impact", IEE Review, Vol. 52, No. 1, $40-44$ (2006).

3. D. Merholz et al.: "Detecting, Tracking and Imaging Space Debris", ESA Bulletin 109, 128-134 (2002). 
4. J. Ender et al. "Radar techniques for space situational awareness," 2011 12th International Radar Symposium (IRS), Leipzig, Germany, 2011, pp. 21-26..

5. S. Samsonov, et al.: "CW Operation of a W-Band High-Gain Helical-Waveguide Gyrotron Traveling-Wave Tube”, IEEE Electronic Device Letters, Vol. 41, No. 5, 773-776 (2020)

6. G. Denisov, et al.: "Gyro-TWTs with Helically Corrugated Waveguides: Overview of the Main Principles", International Conference on Infrared, Millimeter and Terahertz Waves, Paris, France, 1-3 (2019)

7. M. Czerwinski and J. Usoff: "Development of the Haystack Ultrawideband Satellite Imaging Radar", Lincoln Laboratory Journal, Vol. 21, No. 1, 28-44, (2014).

8. M. MacDonald et al.: “The HUSIR W-Band Transmitter", Lincoln Laboratory Journal, Vol. 21, No. 1, 106114 (2014).

9. J. Eshbaugh et al.: "HUSIR Signal Processing", Lincoln Laboratory Journal, Vol. 21, No. 1, 115-134 (2014).

10. S. Samsonov et al.: "Cascade of Two W-Band Helical-Waveguide Gyro-TWTs With High Gain and Output Power: Concept and Modeling", IEEE Transaction on Electron Devices, Vol. 64, No. 3, 1305-1309 (2017).

11. J. Doane: "Propagation and Mode Coupling in Corrugated and Smooth-Wall Circular Waveguides", Infrared and Millimeter Waves, Vol. 13, Ch. 5, 123-170 (1985).

12. J. Doane: "Design of Circular Corrugated Waveguides to Transmit Millimeter Waves at ITER", Fusion Science and Technology, Vol. 53, No. 1, 159-173 (2008).

13. J. Anderson et al.: "Beyond Fusion: The Application of Fusion-Based Microwave Technology to Other Industries", International Conference on Infrared, Millimeter and Terahertz Waves, Paris, France, 1-2 (2019).

14. J. Doane and C. Moeller: "HE11 Mitre Bends and Gaps in a Circular Corrugated Waveguide", International Journal of Electronics, Vol. 77, No. 4, 489-509, 1994.

15. E. Kowalski: "Miter Bend Loss and High Order Mode Content Measurements in Overmoded MillimeterWave Transmission Lines" Master-Thesis, Massachusetts Institutes of Technology (2010).

16. J. Doane: "Grating Polarizers in Waveguide Miter Bends", International Journal of Infrared and Millimeter Waves, Vol. 13, No. 11, 1727-1743 (1992).

17. D. Haas et al.: "Broadband Polarizer Miter Bend for High-Power Radar Applications", German Microwave Conference, Cottbus, Germany, 76-79 (2020).

18. D. Haas, et al.: "Broadband Rotary-Joint Concept for High-Power Radar Applications", European Microwave Conference, Utrecht, Netherlands (2020, accepted).

19. M. Thumm and W. Kasparek: "Passive High-Power Microwave Components", Transaction on Plasma Science, Vol. 30, No. 3, 755-786 (2002).

20. J. Doane: "Parabolic Tapers for Overmoded Waveguides", International Journal of Infrared and Millimeter Waves, Vol. 5, No. 5, 737-751 (1984).

21. https://www.vadiodes.com/en/products/vector-network-analyzer-extension-modules (11. May 2020)

22. P. Clarricoats and A. Olver: Corrugated Horns for Microwave Antennas. IEE Electromagnetic Waves Series 18 (1984)

23. P. Clarricoats et al.: "Propagation and Radiation Behavior of Corrugated Feeds: Part 2, Corrugated ConicalHorn Feed", Proceedings of the Institution of Electrical Engineers, Vol. 118, No. 9, 1177-1186 (1971).

24. J. Doane: "Transitions to Oversized Corrugated Waveguides", International Conference on Infrared and Millimeter Waves, Monterey, USA (1999).

25. http://www.ga.com/transitions-for-testing-high-power-waveguide (11. May 2020)

26. M. Thumm: "Applications of high-power microwave devices". Generation and Application of High Power Microwaves. Vol. 305. Inst. Phys., 1997.

27. M. Thumm et al.: "Computer-Aided Analysis and Design of Corrugated TE11 to HE11 Mode Converters in Highly Overmoded Waveguides”, International Journal of Infrared and Millimeter Waves, Vol. 6, No. 7, 577-597 (1985).

28. A. Ishimaru: Electromagnetic Wave Propagation, Radiation and Scattering: Second Edition. John Wiley \& Sons, 2017.

29. H. Rowe and W. Warters: "Transmission in Multimode Waveguides with Random Imperfactions", The Bell System Technical Journal, Vol. 41, No. 3, 1031-1170 (1962).

30. B. Katsenelenbaum et al.: Theory of Nonuniform Waveguides: The Cross-Section Method, IEE Electromagnetic Wave Series 44 (1998)

31. H. Li and M. Thumm: "Mode Conversion due to Curvature in Corrugated Waveguides" International Journal of Electronics, Vol. 71, No. 2, 333-347 (1991).

32. H. Li and M. Thumm: "Mode Coupling in Corrugated Waveguides with Varying Wall Impedance and Diamter Change", International Journal of Electronics, Vol. 71, No. 5, 827-844 (1991)

33. K. Atkinson, W. Han and D. Stewart: Numerical solution of ordinary differential equations. Vol. 108. John Wiley \& Sons, 2011. 
34. J. Doane: "Mode Converters for Generating the HE11 (Gaussian-Like) Mode from TE01 in a circular Waveguide", International Journal on Electronics, Vol. 53, No. 6, 573-585 (1982).

35. P. Clarricoats et al.: "Propagation and radiation behavior of corrugated feeds: Part 1, Corrugated Waveguide Feed", Proceedings of the Institution of Electrical Engineers, Vol. 118, No. 9, 1177-1186 (1971).

36. G. Bryant and A. Inst: "Propagation in Corrugated Waveguides" Proceedings of the Institution of Electrical Engineers, Vol. 116, No. 2, 203-213 (1969).

37. P. Clarricoats et al.: "Propagation Behaviour of Periodically Loaded Waveguides", Proceedings of the Institution of Electrical Engineers, Vol. 115, No. 59, 652-661 (1968).

38. L. Field: "Some Slow-Wave Structures for Traveling-Wave Tubes", Proceedings of the IRE, Vol. 37, No. 1, 34-40 (1949).

39. E. Chu and W. Hansen: "The Theory of Disk-Loaded Wave Guides", Journal of Applied :Physics 18, 9961008 (1947).

40. C. Chong et al. "Bragg Reflectors", Transaction on Plasma Science, Vol. 20, No. 3, 393-402 (1992).

41. L. Zhang et al.: "Design and Measurement of a Broadband Sidewall Coupler for a W-Band Gyro-TWA", Transaction on Microwave Theory and Techniques, Vol. 63, No. 10, 3183-3190 (2015).

42. E. Nanni et al.: "Low-Loss Transmission Lines for a High-Power Terahertz Radiation", Journal of Infrared, Millimeter and Terahertz Waves, Vol. 33, 695-714 (2012).

43. http://www.ga.com/straight-corrugated-waveguides (11. Mai 2020).

44. I. Lindell and A. Shivola "Dielectrically Loaded Corrugated Waveguide: Variational Analysis of a Nonstandart Eigenproblem", Transaction on Microwave Theory and Techniques, Vol. 31, No. 7, 520526 (1983).

45. K. Zhang and D. Li: "Metallic waveguides and resonant cavities". Electromagnetic Theory for Microwaves and Optoelectronics (2008).

46. D. Pozar: "Microwave Engineering". Fourth Edition, University of Massachusetts at Amherst, John Wiley \& Sons, Inc (2012).

47. W. Gautschi: "Numerical Analysis". Second Edition. Birkhäuser (2010).

48. G. Stoyan and A. Baran: "Elementary Numerical Mathematics for Programmers and Engineers". Birkhäuser (2016).

49. I. Bronstein et al.: Taschenbuch der Mathematik: 10. Auflage, Europa Lehrmittel 2016 (in German)

50. https://de.mathworks.com/help/matlab/ref/eps.html (11. Mai 2020)

51. M. Thumm: "High-Power Mode Conversion for Linearly Polarized HE11 Hybrid Mode Output", International Journal on Electronics, Vol. 61, No. 6, 1135-1153 (1986)

52. M. Thumm et al.: "Design of Short High-Power TE11-HE11 Mode Converters in Highly Overmoded Corrugated Waveguides", Transaction on Microwave Theory and Techniques, Vol. 39, No. 2, 577-597 (1991).

Publisher's Note Springer Nature remains neutral with regard to jurisdictional claims in published maps and institutional affiliations. 